\title{
The Construction of Food Testing Intelligent Laboratory Based on "Internet+" and Big Data Technology
}

\author{
Kai Chen ${ }^{1,2}$, Miao Hao ${ }^{1,2}$, Hong Tan ${ }^{1,2, *}$, Wei Huang ${ }^{2}$, Fangjian Xin ${ }^{2}$ and Bin $\mathrm{Su}^{2}$ \\ ${ }^{1}$ Guizhou Academy of Testing and Analysis, Guiyang, Guizhou, 550000, China \\ ${ }^{2}$ Guizhou Academy of Sciences Big Data Co.LTD, Guiyang, Guizhou, 550000, China
}

\begin{abstract}
Food safety testing is of great significance for building a harmonious society. With the advent of the Internet era, the number of data indicators to be considered in food safety testing has gradually increased, and traditional food testing cannot adapt to the fast pace of today's times. In order to meet the current challenges of food testing, this paper analyses the advantages of "Internet + " in building a food testing laboratory based on the challenges of food safety testing in the Internet era, and discusses the advantages of "Internet $+"$ in the era of food testing. The paper discusses the intelligent working mode of food testing laboratories in the "Internet + " era, and combines big data technology to build an intelligent and comprehensive laboratory management platform, so as to build a new era of food testing intelligent laboratory, which improves the efficiency of food testing managers, reduces the workload, improves the accuracy of food safety testing, and helps the healthy development of the food industry.
\end{abstract}

\section{Introduction}

Food quality concerns the lives and health of the citizens of society at large, and any food safety issue may cause social concern, so food testing laboratories that test for food safety issues have an important role in the field of food safety. Food safety, is necessary to maintain our social stability, economic and foreign trade development, and ultimately create a harmonious society. The food inspection and testing laboratories, the main focus of work on the inspection and testing of food safety, it plays an important role in determining. With the continuous progress of science and technology, society for food testing objective conditions put forward higher standards, and the existing manual management model system under the work of some laboratories, has been less adapted to the development of today's advanced productivity requirements. Based on the concept of "Internet +", advanced food testing laboratories at home and abroad began to gradually introduce efficient network management mode. And with the deepening of laboratory quality management, the increasing amount of testing data will inevitably bring about management oversight, so there is an urgent need to introduce effective data management methods to effectively manage the data. Therefore, this paper addresses the shortcomings of current food testing laboratories, and introduces big data technology to build a food testing intelligent laboratory, taking into account the background of the "Internet+" era, to promote the further development of food safety research [1-2].

\section{Intelligent laboratory for food testing in the context of Internet era}

\subsection{Feasibility analysis}

The "Internet $+"$ is a practical concept developed gradually in the Internet era, which promotes the combination of traditional industries and the Internet based on the advantages of information technology and platforms for the deep integration of the Internet and traditional industries, bringing into play the common advantages of the two. Food testing can also benefit from the "Internet + ", through the construction of intelligent laboratory, food testing effectiveness can be improved.

Intelligent laboratory is the "Internet + " thinking into the laboratory construction and management, uphold the "people-oriented" basic concept. In technology to achieve intelligence and information technology, focusing on the improvement of efficiency. The creation of food testing intelligent laboratory is in the food laboratory construction and management of technology upgrades, so that the laboratory operation more efficient, safe and convenient. In the context of the current national concern for the health of citizens, with the progress of science and technology, the introduction of "Internet + " to build a food testing intelligent laboratory has a practical feasibility [3].

\footnotetext{
*Corresponding author: tanh@gzbdi.com
} 


\subsection{Advantage Analysis}

The "Internet +" emphasizes the advantages of the Internet, and the construction of intelligent laboratories can make the relevant advantages intuitive. In the case of food testing, the Internet information sharing pool can provide massive resources to help the laboratory work. For example, the common big data technology can be used to collect the standard parameters of the object target when conducting food testing, and then match the standard parameters with the results of food testing to obtain scientific conclusions. In addition, the richness of Internet resources is also conducive to the updating of information in the intelligent laboratory. The intelligent laboratory mainly relies on rich information resources for its work, and in the era of "Internet +", these resources can be quickly collected in the laboratory to play a long-term positive role.

The key to food testing is the scientific value of the results, and the integration of traditional testing and "Internet +" based on intelligent laboratories is conducive to improving the scientific nature of testing. Conventional testing work mainly relies on personnel and equipment, instruments, in the testing process, operational errors, equipment aging and other issues may affect the scientific nature of testing, accuracy, based on intelligent laboratory, a lot of work can be automated, effectively avoiding errors in personnel operations, but also to avoid the adverse effects of aging equipment, the accuracy of food testing is guaranteed.

\section{Intelligent laboratory design based on big data technology}

\subsection{Overall Architecture}

Intelligent laboratory is based on the Internet + , which uses the big data technology to conduct comprehensive intelligent perception and monitoring. The whole system is based on big data technology, and constructs an intelligent integrated management platform to realize the intellectualization, safety and visualization management of the whole laboratory, ensure its safety, and create a green, low carbon, low consumption, intelligent and safe experimental environment. User-user, user-laboratory, user-information resources, laboratory-laboratory information interaction are all done by the intelligent of the laboratory system, no longer need the intervention of the experimenter, which is the perfect state of the intelligent of the

The intelligent lab system is built on the basis of Internet of Things, and mainly consists of a three-layer structure of physical layer, communication layer and application layer. The physical layer is responsible for collecting and sending the data such as environmental variables, item status and alarm information of the lab to the communication layer. The communication layer sends the packaged data from the physical layer to the Internet via GPRS network. The server of the application layer is responsible for receiving and processing the received data and verifying, unpacking, parsing, processing and storing them. The data center is responsible for processing the data stored in the server database and then presenting the results. The overall architecture of the whole lab is shown in Fig.1.

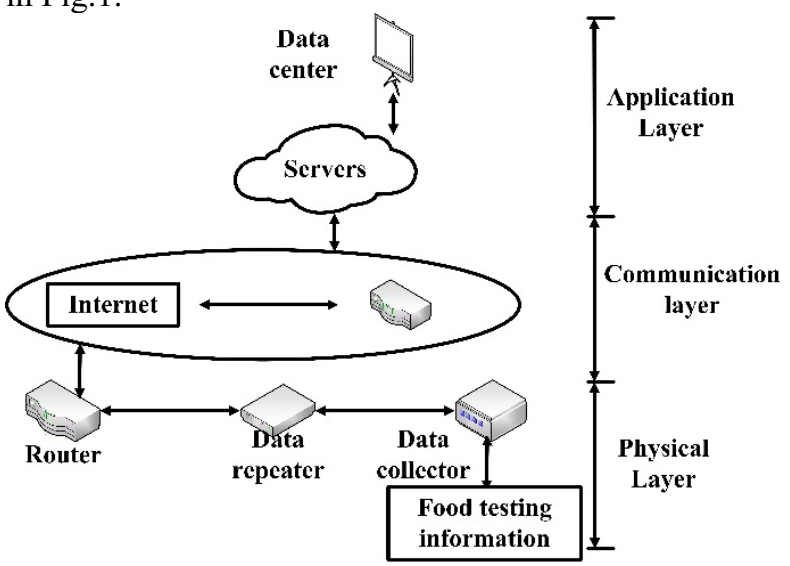

Figure 1. Overall architecture of the whole lab

\subsection{Management of food testing information}

A lot of food testing information needs to be processed in food testing intelligent laboratory, including personnel information, supplier information, customer information, inspection subcontracting information, equipment information, reagent consumables information, standard information, sample information and inspection information, as shown in Fig.2.

\section{Food testing information}

Testing personnel information

Supplier information

Inspection subcontractor information

Equipment information

Reagent consumables information

Standard information

Sample information

Inspection information 


\subsection{Application of big data technology in intelligent laboratory}

In the process of food testing, food testing information can be reflected by data. Data processing and analysis is to analyse massive food testing data. Data specification is the basis and tool of data analysis. Only by establishing practical data rules and unifying and standardizing the laboratory testing data, can we obtain correct food testing results and explore valuable food safety information.

In order to realize the analysis of massive food testing data, so as to effectively grasp the safety situation of the detection, a large number of data need to be stored and managed. Therefore, in order to manage and transfer the required data very conveniently, accurately and quickly, a good data organization structure and database must be established as a key indicator to measure the operation of the laboratory.

The data warehouse is different from the traditional database. The database is a large number of data sets that have not been sorted out, and the data warehouse is a collection of systematic databases that have been sorted out, planned and constructed from the database. The data contained in data warehouse is clean, complete and integrated. The smooth establishment and efficient operation of data warehouse is the basis of data analysis and mining valuable information.

In the process of building food testing intelligent laboratory, in order to ensure the efficiency of food testing data analysis results, we need to establish a food safety data warehouse for daily food safety testing data to realize real-time monitoring and early warning of the overall state of food safety. Through statistical analysis and data mining, we can evaluate the state of food safety, analyse and predict the development trend of food safety, and provide scientific decision-making basis for food safety management and control. The main technical framework and process of data warehouse are as follows.

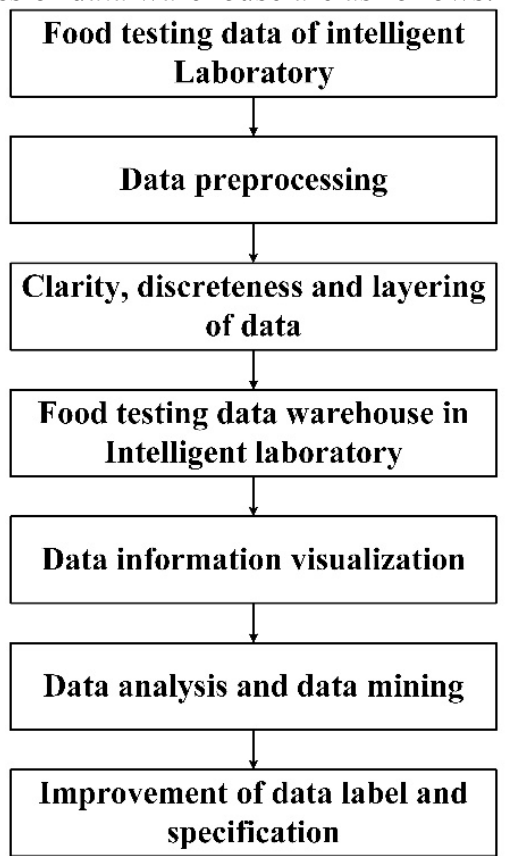

Figure 3. The main technical framework and process of data warehouse

\subsubsection{Data processing}

The database that stores these test data usually contains the following information: food name, type, origin, sample number, test time, test items, test results and units, import and export countries, production enterprises and so on. Data analysis mainly focuses on the types of food, origin, testing time, testing items and results. In order to process the data in the database, these data need to be extracted separately before data analysis.

\subsubsection{The establishment of data discrete layered rule}

The data of food and agricultural products testing in recent 10 years were collected and sorted. Through the methods and steps of data cleaning, data conversion was carried out, data labels and cleaning rules were established, and a series of data dictionaries of food testing data were formed, including item dictionaries, item dictionaries. A complete set of concept hierarchy and coding rules system for key attributes of laboratory food and agricultural products testing data, such as product dictionary table and country and region dictionary table.

\subsubsection{Application of food testing data warehouse}

The fundamental purpose of food testing data warehouse is to support decision-making, that is to provide data information support for improving the efficiency of inspection supervision and decision-making. High level risk analysts, middle-level business processing and supervision personnel and grass-roots laboratory testing personnel and other different levels of management personnel can use the data warehouse for decision-making analysis to improve the quality of management decisions [4-6]. The data warehouse architecture is shown in Fig.4.

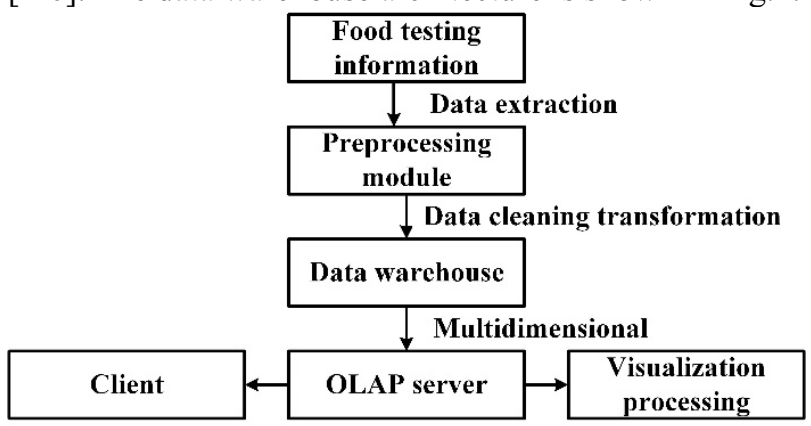

Figure 4. The data warehouse architecture

A large amount of testing data of food testing intelligent laboratory is effectively used through data warehouse, which realizes the intuitive analysis of testing situation and detection situation. In order to realize the functions of information processing, report presentation, early warning and auxiliary decision-making, it has the characteristics of dynamic, comprehensive and humancomputer system, and integrates the laboratory testing data in food testing into an organic whole, The reliability and accuracy of data analysis are realized, and the work efficiency is improved. In the process of building data warehouse, we use data mining tools to extract value information, and use scientific data mining algorithms, such as association rules, cluster analysis, automatic 
prediction and so on. Based on the establishment of food testing data warehouse, valuable information is explored to help analyse the food safety situation, and analysis conclusions are given in a more intuitive and easy to understand form, so as to grasp the law of food safety change, predict the development trend, and better improve the scientificity and predictability of testing.

\section{Conclusion}

With more and more attention paid to food safety, food detection has also been paid attention to by the society. In order to effectively improve the efficiency and accuracy of food inspection, this paper builds a food testing intelligence laboratory Based on "Internet + " and big data technology, this paper analyses the feasibility and advantages of the intelligent laboratory, designs the overall architecture of the laboratory, and designs the corresponding data warehouse for large amounts of food detection information by introducing big data technology. The results of this paper can improve the efficiency and accuracy of food detection and promote the further development of food safety detection.

\section{Acknowledgement}

This work was supported by Guizhou Provincial Science and Technology Projects ([2015]4005) and Guizhou Provincial Science and Technology Projects ([2018]5404).

\section{References}

1. L. Boschis et al., "Smart micro-sensing: Antibodies and aptamer-based micro-ELISA as performing offline/on line tool for allergens and mycotoxins detection in foods," 2017 IEEE Biomedical Circuits and Systems Conference (BioCAS), Turin, Italy, 2017, pp. 1-4.

2. S. N. Nihtianov, G. C. M. Meijer and K. M. M. Kerkvliet, "New methods for non-destructive sterility testing of aseptically packed food products," IMTC/98 Conference Proceedings. IEEE Instrumentation and Measurement Technology Conference. Where Instrumentation is Going (Cat. No.98CH36222), St. Paul, MN, USA, 1998, pp. $537-$ 540 vol.1.

3. Z. Wang, C. Chen, B. Guo, Z. Yu and X. Zhou, "Internet Plus in China," in IT Professional, vol. 18, no. 3, pp. 5-8, May-June 2016.

4. H. L. H. S. Warners and R. Randriatoamanana, "Datawarehouser: A data warehouse artist who have ability to understand data warehouse schema pictures," 2016 IEEE Region 10 Conference (TENCON), Singapore, 2016, pp. 2205-2208.

5. O. Baker and C. N. Thien, "A New Approach to Use Big Data Tools to Substitute Unstructured Data Warehouse," 2020 IEEE Conference on Big Data and Analytics (ICBDA), Kota Kinabalu, Malaysia, 2020, pp. 26-31.
6. B. K. Seah and Nor Ezam Selan, "Design and implementation of data warehouse with data model using survey-based services data," Fourth edition of the International Conference on the Innovative Computing Technology (INTECH 2014), Luton, UK, 2014, pp. 58-64. 